\title{
Importancia del microscopio en el análisis de pelos en la Criminología y en la Criminalística
}

\author{
The Importance of the Microscope in Hair Analysis \\ in Criminology and Criminalistics \\ D. C. Norma Elena Rojas Ruíz \\ Libre de Estudios Universitarios Licenciatura en Criminología, Criminalística y \\ Técnicas Periciales \\ D. C. Guillermo Muñoz Zurita \\ Facultad de Medicina de la Benemérita Universidad Autónoma de Puebla \\ (FMBUAP) \\ Alejandro Cruz Galaviz \\ Libre de Estudios Universitarios Licenciatura en Criminología, Criminalística y \\ Técnicas Periciales
}

Fecha de recepción del artículo Febrero de 2012

Fecha de aceptación del artículo

Febrero de 2012

Introducción: El uso del microscopio en el análisis del pelo como evidencia forense en una escena de crimen, sigue siendo una herramienta de suma importancia, ya que de manera macroscópica es imposible evidenciar características propias del pelo, tales como la presencia o ausencia de médula, pigmentación, patrón que exhibe el canal medular, el cual permite determinar la posible especie animal a la que pertenece. Mediante la observación al microscopio también es posible el hallazgo de elementos que contribuyan al esclarecimiento del hecho delictuoso, tales como adherencias ó inclusiones, las cuales podrían estar relacionadas con la víctima o el agresor. Material y Método: En el presente artículo se analizaron al microscopio diferentes muestras de pelo de origen animal y humano, con la finalidad de observar sus características microscópicas. Resultados: Se observaron diferencias en los elementos pilosos de acuerdo al origen animal o humano, sobre todo en el canal medular y la cantidad de pigmento que tenía el elemento piloso.

Conclusiones: El uso del microscopio permite la observación de las características diferenciales entre elementos pilosos de origen humano y animal, por lo cual es una herramienta indispensable en el proceso de la investigación en criminalística. 


\section{Palabras claves}

Pelo, médula, canal medular, Criminalística .

\section{Summary}

Introduction: The use of the microscope in hair analysis as forensic evidence at the scene of the crime continues to be an extremely important tool. It is macroscopically impossible to identify characteristics of hair, such as the presence or absence of medulla, pigmentation, medullary canal pattern, which permit determination of which animal species the hair belongs to. By using the microscope, it is also possible to find elements which contribute to explain a criminal event, such as adherences or inclusions, which could be related to the victim or aggressor.

Material and Method: In the present article, different samples of animal and human hair were analyzed by microscope, in order to observe their microscopic characteristics.

Results: Differences were observed in hair elements according to animal or human origin, especially in the medullary canal and the quantity of pigment the hair element had.

Conclusions: The use of the microscope allows the observation of characteristic differences between hair elements of human and animal origin; it is therefore an indispensable tool in the criminalistic investigation process.

\section{Key words}

Hair, medulla, medullary canal, criminalistics.

\section{Introducción}

El pelo y los vellos son constituyentes importantes presentes en el cuerpo de los animales mamíferos, son una característica que permite diferenciarlos del resto de los animales. Generalmente estamos acostumbrados a observar el pelo sólo de forma macroscópica, sin embargo el pelo guarda información importante que nos define como individuos pertenecientes a cierto tipo de raza, género biológico, e incluso puede proporcionarnos información acerca del estado nutricional o la ingesta de sustancias tóxicas. Actualmente el análisis de los pelos y fibras encontrados como evidencia en escenas de hechos delictivos, proporciona información de suma importancia para el esclarecimiento del mismo. Incluso al observar el pelo como evidencia al microscopio, hace evidente el hallazgo de características que a simple vista sería imposible, tales como la presencia de adherencias de otros materiales como grasa, metales, arena, o simplemente la observación de sus constituyentes En el campo de la criminalística los pelos y fibras constituyen indicios de tipo microscópico, por lo cual su análisis en el laboratorio es típicamente mediante el uso del microscopio de luz la cual involucra dos pasos fundamentalmente: la identificación del cabello y su comparación con cabellos de origen conocido, esto permite determinar si corresponden o no con el comparado (1).

El pelo bioquímicamente está constituido principalmente de fibras de proteí- 
nas como la queratina, las cuales son responsables de su estructura (2) y pigmentos. Estas características son más que solo cuestiones químicas, ya que permiten reconocer diferencias significativas entre pelos correspondientes a varias razas, origen del pelo (parte corporal a la que pertenece), e incluso los cambios en las características microscópicas hacen evidente si su caída fue de manera normal como parte de la muerte del pelo o si fue arrancado con violencia, lo cual es de suma importancia en la criminalística.

A finales del siglo pasado, el análisis de pelo era usado principalmente para evaluar la exposición a metales pesados y solo a principios de los 80 se realizaron las primeras investigaciones en el campo de la toxicología forense, encontrando las primeras concentraciones de drogas de abuso en el pelo (3). Debido a que el pelo es una muestra en la cual es fácil la conservación de diversas sustancias, ha favorecido su estudio en el campo de la Criminalística y la Medicina Forense, otra característica importante es el hecho de que su recolección sea sumamente sencilla, incluso a diferencia de otras muestra biológicas, el pelo no puede ser alterado físicamente, por lo tanto es imposible manipular los resultados de análisis practicados. Para el caso de las drogas, el pelo provee una ventana más amplia de detección que muestras como orina y sangre (del orden de varias semanas a meses) en las cuales las drogas permanecen durante un corto periodo $(3,4)$.

Entre los estudios de detección de drogas en pelo humano, se realizó un estudio para detectar cocaína en muestras de pelo de reclusos bajo régimen abierto adscritos al Centro Francisco Canestri, Caracas, mediante pruebas de Inmuno ensayo Competitivo Rápido (IC), Espectrofotometría de Luz Ultravioleta (LUV) y Cromatografía en Capa Fina, sus resultados demostraron que con las metodologías de IC y LUV, es posible la detección de cocaína en pelo, pero con la CCF no fue posible la detección de la droga, ya que se requiere mayor concentración de la sustancia (5).

En el presente estudio se analizaron las características vistas al microscopio que, exhiben muestras de pelo de distinto origen animal y humano, y se resalta la importancia hasta el día de hoy del microscopio en el análisis de elementos pilosos.

\section{Materiales y métodos}

Se elaboraron preparaciones microscópicas permanentes en Portaobjetos 26 x $76 \mathrm{~mm}$ de muestras de pelo humano de diferentes partes del cuerpo: cabeIlo, ceja, barba, vello púbico, así como muestras de pelo de diferentes animales, vaca, cerdo, perro, caballo. Las muestras fueron observadas a los aumentos de 4X,10X, 40X y 100X de un microscopio compuesto marca Leica DM500. Se tomaron microfotografías con una cámara Digita de 14 MP. Las muestras fueron analizadas por la presencia o ausencia de médula, patrón medular exhibido, pigmento en la corteza, presencia o ausencia de escamas en la cutícula, presencia o ausencia de bulbo en el elemento piloso, si tenía bulbo se describió la fase de crecimiento en que se encontraba. Con las preparaciones microscópicas obtenidas se elaboró un catálogo.

\section{Resultados y discusión}

La importancia del análisis de pelos y fibras en el campo de las ciencias forenses ha cobrado actualmente gran importancia, ya que de acuerdo con los principios de la criminalística, es posible que ocurra un intercambio de evidencia física entre la víctima y el victimario. Debido a esto es fundamental el análisis de las características a nivel microscópico que pueden presentar los diferentes elementos pilosos. 
Con respecto al análisis del pelo humano se observaron diferencias dependiendo de la parte corporal a la que pertenecía (Figura 1 y 2 y tabla 1).

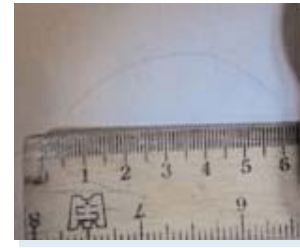

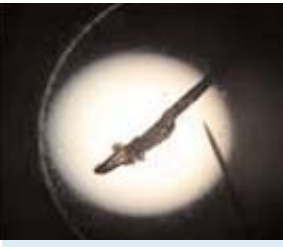

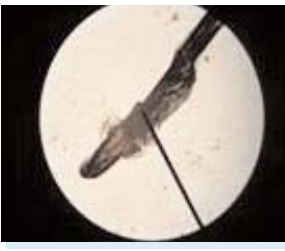

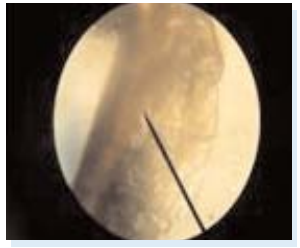

Figura 1. a) Cabello humano observado a simple vista, Las siguientes figuras pertenecen al mismo cabello humano observado al microscopio compuesto en diferentes aumentos:
b) aumento de $10 X$
c) aumento de $10 \mathrm{X}$
d) aumentado en 40X.
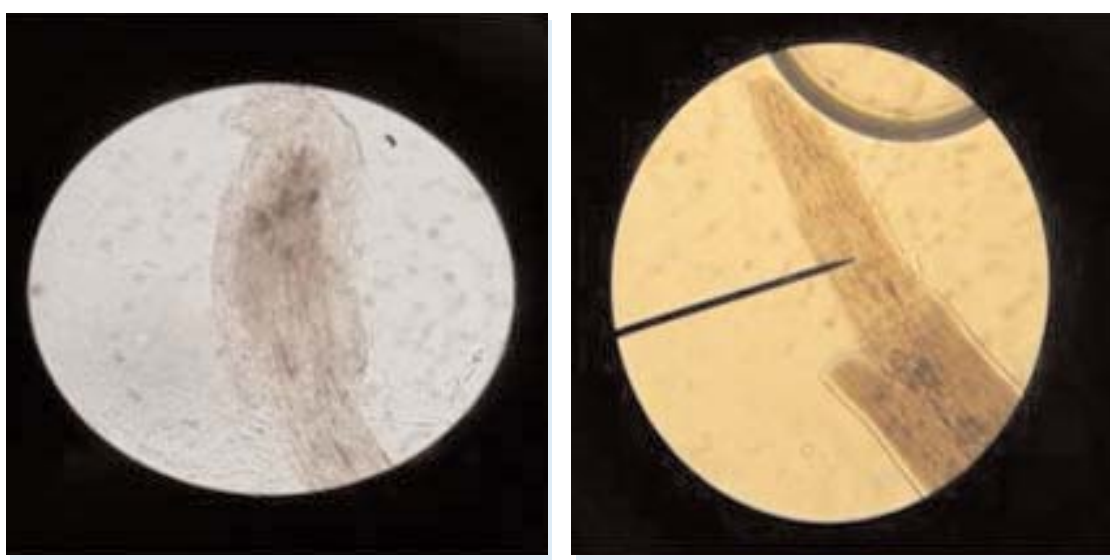

b

Figura 2

a) Ceja humana observado al microscopio con un aumento de $40 \mathrm{X}$

b) Pelo axilar observado con un aumento de 40X presenta ruptura por estrés mecánico.

Tabla 1

Descripción de las características microscópicas del pelo humano de acuerdo a su región corporal

\begin{tabular}{|l|l|l|}
\hline Tipo de pelo & Humano & Humano \\
\hline $\begin{array}{l}\text { Región } \\
\text { corporal }\end{array}$ & Ceja & Axilar \\
\hline Color & Castaño claro & Castaño medio \\
\hline Bulbo & Etapa anágena & Sin bulbo \\
\hline $\begin{array}{l}\text { Tamaño } \\
\text { pantidad de } \\
\text { pigmento }\end{array}$ & Muy poca melanina & $\begin{array}{l}\text { Con suficiente } \\
\text { melanina }\end{array}$ \\
\hline $\begin{array}{l}\text { Punta extremo } \\
\text { distal }\end{array}$ & Cónica & $\begin{array}{l}\text { Desgarrada por estrés } \\
\text { mecánico }\end{array}$ \\
\hline
\end{tabular}


Estas diferencias radican en la cantidad de pigmento que tiene el elemento piloso la cual en la muestra observada es mayor en el cabello, con respecto a la ceja y axila. La fase de crecimiento del cabello humano y la ceja se encontraban en fase anágena. En el caso de la axila, el elemento piloso no presentaba bulbo debido a un traumatismo, el cual es evidente gracias al uso del microscopio. Esta observación es importante sobre todo en el caso de elementos pilosos que hayan sido arrancados con violencia.

El hallazgo de ciertos elementos pilosos puede estar relacionado al tipo de delito cometido, en los casos de violación o abuso sexual, por ejemplo a la presencia de vellos púbicos, el análisis de estos contribuyen a la posible identificación del agresor. Uno de los estudios realizados en vello púbico de alumnos de la Escuela Médico Militar, concluyó que la probabilidad de que coincidan todas las características de dos vellos púbicos pertenecientes a dos individuos distintos es del 0\% (6). Estos hallazgos hacen evidente la exactitud de las técnicas de análisis de pelos, en este caso vellos púbicos, a la vez hace latente su importancia en la criminalística y las técnicas periciales.

En este estudio se analizaron también pelos correspondientes a diferentes animales, algunos que conviven con el hombre de manera habitual como perros y gatos, otros que se usan para fines alimenticios como ganado vacuno y caprino. Se realizó esto debido a que en algunas ocasiones pueden ser encontrados pelos de animales en ropa de víctimas, en cuyo caso se consi-

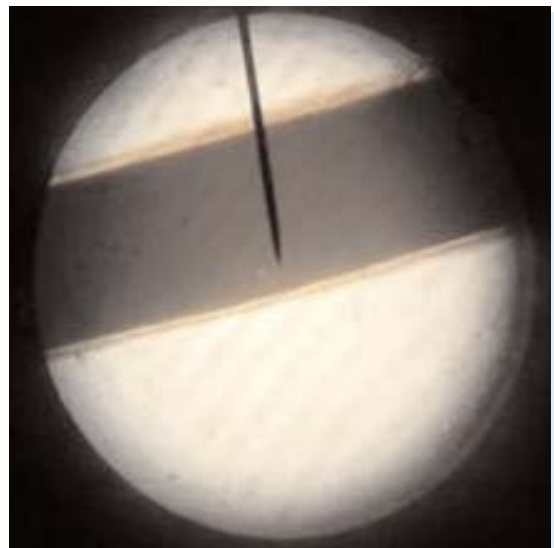

a

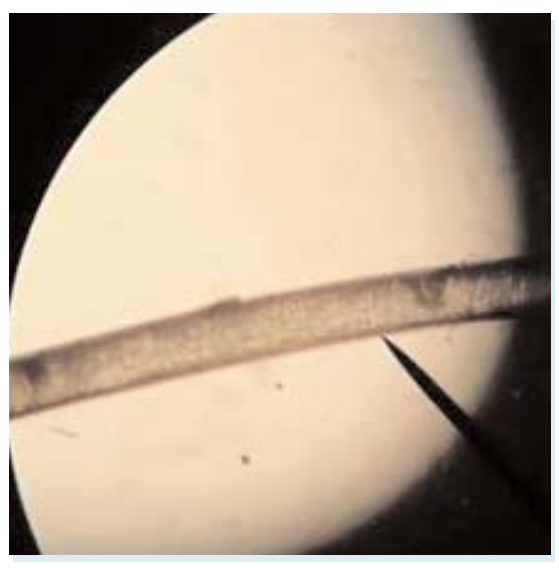

c

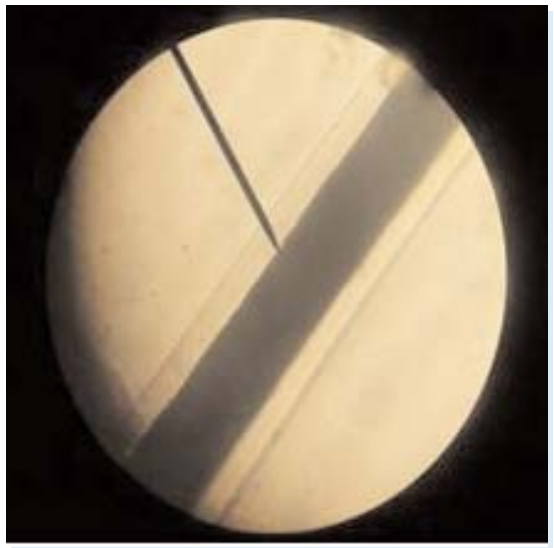

b

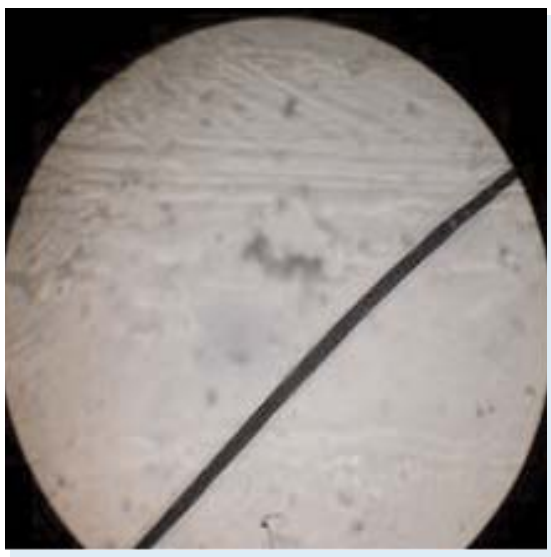

d

Figura 3

a) Pelo de vaca observado al microscopio con un aumento de $40 \mathrm{X}$

b) Pelo de caprino observado con un aumento de $40 \mathrm{X}$

c) Pelo de cola de caballo observado con un aumento de $40 \mathrm{X}$

d) Pelo de perro observado con un aumento de $10 \mathrm{X}$ 
dera que el pelo animal llego por transferencia, y que podría tener una posible relación con la víctima o el agresor.

Al realizar el análisis al microscopio del pelo de origen animal, se observó de manera evidente las diferencias microscópicas con respecto al pelo humano, por ejemplo la presencia de médula continua en la mayoría de animales y un patrón en el canal medular que varía dependiendo de la especie, ya que en el caso del pelo de vaca, se observa un patrón de canal medular uniseriado que abarca, la mayor parte del diámetro del elemento piloso, en el pelo caprino, es posible observar la corteza con menor cantidad de pigmento, en cambio en el pelo de cola de caballo, no se observa la presencia de médula, por último el pelo de perro mostró la presencia de médula continua, con una gran cantidad de pigmento. (Figura 3).

Esto hace evidente las diferencias a nivel microscópico que exhiben los elementos pilosos, dependiendo del origen animal al que correspondan, incluso se puede distinguir entre pelos pertenecientes a animales emparentados genéticamente.

En un estudio donde se analizaron muestras de pelo del lobo Ibérico y del perro, se identificaron diferencias significativas a nivel microscópico el número de bandas por pelo entre ambas especies. (7)

\section{Conclusiones}

El análisis del pelo humano mediante el uso del microscopio sigue siendo una parte fundamental de la investigación en la Criminalística, ya que hace evidente los componentes a nivel microscópico de la estructura del pelo, así como las diferencias con respecto al pelo de origen animal. Además con el reciente avance en las técnicas de Biología Molecular será posible la búsqueda de evidencias en el pelo, que permitan encontrar la respuesta a hechos delictuosos.

\section{Bibliografía}

1. 1. Deedrick, D.W. 2000. Hairs, Fibers, Crime, and Evidence. Forensic Science Communications. V. 2. No 3.

2. Mathews, C.K. and K.E.Van Holde.1998. Bioquímica. Ed. McGraw Hill- Interamericana. PP. 189-191.

3. Valdebenito Zenteno, G. y M. E. BÁez Contreras. 2007. El pelo: ¿esconde secretos para LA CIENCIA FORENSE?.CiENCIA...AHORA. 20: 103-110.

4. Kintz, P. 2004. Value of hair analysis in Postmortem toxicology. Forensic SCience INTERNATIONAL. 142: 127-134.

5 díaz Tremarias, M. 2003. Detección de Cocaína en Pelo y Orina de Reclusos bajo

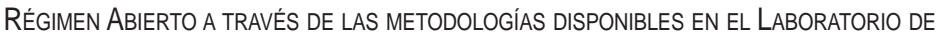
Toxicología, Escuela de Bioanálisis, UCV. Retel ReVISTA de toxicología en línea. Pr. 2438.

6. HeRnÁNDEZ-ChÁVEZ, J.R. 2006. ESTUdio MICROSCóPICO DEL VELLO PÚBICO HUMANO Y SU utilidad PARA LA IDENTIFICACIÓN FORENSE. Rev SANid Milit MeX. 60(5): 297-303.

7. Porta X. y L. Llaneza. 2001. Diferencias macroscópicas entre pelos de PeRro (CANiS FAMILIARIS LINNAEUS, 1758) Y Lobo IbÉRICo (CANIS LUPUS SIGNATUS CABRERA, 1907). GALEMYS. 13: 205-215.

Correspondencia

Guillermo Muñoz Zurita FMBUAP

Calle 13 sur 2702

Colonia Volcanes C.P 72000

Tel 2295500 Ext.6061 Puebla Puebla (México) guimuzu2003@yahoo.com 